\title{
Impacts of human activities on dioxins emissions at national scale
}

\author{
Zhiyuan Ren, Minghui Zheng* \\ State Key Laboratory of Environmental Chemistry and Ecotoxicology, Research Center for Eco-Environmental Sciences, Chinese Academy of Sciences, P.O. Box 2871,
} Beijing 100085, China

\section{A R T I C L E I N F O}

\section{Article history:}

Received 23 September 2008

Received in revised form 18 March 2009

Accepted 24 March 2009

Available online 8 May 2009

\section{Keywords:}

PCDD/PCDFs

Human activities

HDI

Inventory

Industry

Sources

\begin{abstract}
A B S T R A C T
In this study, the dioxins emissions from 51 countries were investigated by multivariate statistical analysis, and the correlations between dioxins emissions and some aspects of human activities were discussed at national scale.

For the 51 countries mentioned in this paper, the total emissions are $36.0 \mathrm{~kg}-\mathrm{TEQ} \mathrm{yr}^{-1}$, and the average values of annual dioxins emissions (from 2000 to 2007) per capita and per unit GDP are calculated to be 18.3 g-TEQ million-people ${ }^{-1} \mathrm{yr}^{-1}$ and $6.7 \mathrm{~g}$-TEQ billion-USD ${ }^{-1} \mathrm{yr}^{-1}$, respectively. Furthermore, as the three leading industrial emission sources of dioxins, power generation and heating, ferrous and non-ferrous metal production and waste incineration contribute $45.1 \%$ to the total emission in all. It is also worthy to note that the uncontrolled combustion processes release about $40 \%$ of the total emission. From the correlations between the dioxins emissions and the factors of human activities, population and land area could be tentatively suggested to be positive indicators to dioxins emissions. Specially, GDP per capita would roughly be a negative indicator to the emissions from the uncontrolled combustion processes, and GDP would roughly be a positive indicator to emissions from the other sources.
\end{abstract}

(ㄷ) 2009 Elsevier Ltd. All rights reserved.

\section{Introduction}

As people highly upgraded the capacity to change the world after industrial revolution, the influences of human activities to the environment are boosted, and the physical environment is therefore facing unprecedented risks. Chemicals, from one aspect, played an important role as a medium for the environmental crisis. It has been commonly acknowledged that to reduce the production and to limit the usage of toxic chemicals are necessary to decrease the negative impact to the environment. Although the industrial production of the toxic chemicals could be strictly controlled, unintentionally produced chemical pollutants are very hard to deal with. Dioxins (including PCDDs and PCDFs), with potential risk to ecosystem and human health at trace level, is one of the typical unintentionally produced persistent organic pollutants (UP-POPs). It could be widely detected in environment and in biological tissues (Dyke et al., 1997; Alcock et al., 2001; Jurado et al., 2004).

Human activities, concerning the industrial production processes, domestic behaviors, and other activities where dioxins may be formed and released, were commonly recognized as the major causes of the dioxins pollution. At national scale, human activities could be described from the aspects of population, economy and development. Needless to say, some indicators of human activities, e.g. population and GDP, might have significant influ-

\footnotetext{
* Corresponding author. Tel.: +86 10 62849172; fax: +86 1062923563 .

E-mail address: zhengmh@rcees.ac.cn (M. Zheng).
}

ences on the dioxins emissions or serve as indicating factors for dioxins emissions.

Due to the toxicity, environmental persistence and potential ecological risk of dioxins, many investigations on the estimation of dioxins emissions have been done at national scale since 1980s (Sheffield, 1985; Fiedler and Hutzinger, 1992; Harrad and Jones, 1992; Eduljee and Dyke, 1996; Gribble, 1996). Nowadays, the inventory methodology is commonly recognized and widely used to estimate dioxins emissions in many countries. After 2002, due to the obligation of the Stockholm Convention ("Convention") on POPs, many parties make up their national dioxins emissions inventories. Up to 2006 , more than 50 countries had compiled their inventories on dioxins emissions, and the numbers of the national inventories are continuously increasing.

By the "Standardized toolkit for identification and quantification of dioxin and furan releases" (abbreviated as toolkit), the dioxins emission sources were suggested to be classified into categories in the inventory, and thus more information about dioxins emissions could be provided in detail. Several thermal processes of industry were confirmed to be important sources of dioxins emissions in previous studies (Quass et al., 2004). The Convention suggested that waste incinerators, cement kilns firing hazardous waste, production of pulp using chlorine and thermal processes in the metallurgical industry have potential for comparatively high emissions to the environment (Stockholm Convention, 2001). In the past years, several developed countries largely reduced the dioxins emissions from some industrial sources (Douben, 1997; Japan, 2005; UK, 2007). 
In this study, the dioxins emission inventories of more than 50 countries are collected and analyzed with the reference years between 2000 and 2007. The multiple dimensional distributions of national dioxins emissions are generated, and the associations between human activities and dioxins emissions are studied on the aspects of economy, population, human development, etc. The results could not only provide the macroscopical information for the decision makers but also reveal some internal impacts of human activities on the dioxins emissions.

\section{Methods and materials}

\subsection{Inventory methodology}

In the 1980s, scientists began to estimate the national dioxins emissions by the inventories (Sheffield, 1985). The inventory methodology has been gradually adopted by many countries to estimate annual dioxins emissions. After the continuous improving and updating in these years, inventory methodology is now used as a reliable tool to estimate the dioxins emissions sorted by emission sources and emission media. To assist the parties to establish their dioxins inventories, the first and the second edition "Toolkit" were successively published in 2003 and in 2005 (UNEP, 2005).

In the inventories, dioxins are calculated and presented in grams of TEQ. Rooting from different schemes of TEFs, there are three types of TEFs accordingly, i.e. N-TEF, I-TEF and WHO-TEF. The previous studies showed that TEQs calculated from different schemes of TEFs might cause the differences of no more than $28 \%$ (Wittsiepe et al., 2007; Bhavsar et al., 2008), whereas these differences could not significantly increase the variations of the data in the inventories. Therefore, the series of TEFs are not specified in this study.
In general, the emission inventory is a matrix divided into nine categories of emission sources in rows and five general emission media in columns. In the present study, the emission media of both residue and soil are combined into one column due to their similarities (Table 1). The categorized emissions are calculated as follows:

Source Strength $=\sum$ Emission Factor Media $\times$ "Activity Rate"

\subsection{Data sources of emission inventories}

The emission data used in this article were mainly collected from three kinds of resources, i.e. National Implementation Plans (NIPs), national inventories on UNEP Chemicals Branch website, and the published literatures.

The dioxins emissions reported in NIPs are mainly between 2002 and 2007. Most of the dioxins inventories in NIPs are reasonable. However, there are still some abnormal data, such as Ivory Coast's (illogical, air), Nicaragua's (illogical, uncontrolled combustion processes) and Chad's (unbelievable, air). UNEP Chemicals (UNEP, 2000-2003) provided 10 inventories from five Asian countries and five Latin American countries, the reference years of these inventories being around 2000. For the collected literatures, the articles reported 24 national inventories with the reference year between 1999 and 2004 (Quass et al., 2004; Breivik et al., 2006; Fiedler, 2007; Van der Gon et al., 2007), and these inventories are mainly from Asia, Europe and Latin American countries.

During the terms of data handling, some of the inventories from these data sources are found to be inconsistent with each other. To resolve this problem, the most logical, the most comprehensive, and the latest inventories are chosen to be our primary data

Table 1

The national emissions inventory for dioxins in Australia, China and Rwanda (unit: g-TEQ $\mathrm{yr}^{-1}$ ).

\begin{tabular}{|c|c|c|c|c|}
\hline & Air & Water & Products & Land or residue \\
\hline \multicolumn{5}{|l|}{ Australia (2004, HDI: 0.863) } \\
\hline Waste incineration & 94.39 & 0.36 & & 30.60 \\
\hline Ferrous and non-ferrous metal production & 112.25 & & & 44.80 \\
\hline Power generation and heating & 34.90 & & & 31.80 \\
\hline Production of mineral products & 1.48 & & & \\
\hline Transport & 8.90 & & & \\
\hline Uncontrolled combustion processes & 240.00 & & & 1020.00 \\
\hline Production and use of chemicals and consumer goods & 0.40 & 0.20 & & 103.00 \\
\hline Miscellaneous & 0.30 & & & 0.85 \\
\hline Disposal and landfill & & 2.60 & & 40.30 \\
\hline Total & 492.62 & 3.16 & 0.00 & 1271.35 \\
\hline \multicolumn{5}{|l|}{ China (2004, HDI: 0,768) } \\
\hline Waste incineration & 610.47 & & & 1147.10 \\
\hline Ferrous and non-ferrous metal production & 2486.20 & 13.5 & & 2167.20 \\
\hline Power generation and heating & 1304.40 & & & 588.10 \\
\hline Production of mineral products & 413.61 & & & \\
\hline Transport & 119.70 & & & \\
\hline Uncontrolled combustion processes & 64.00 & & & 953.00 \\
\hline Production and use of chemicals and consumer goods & 0.68 & 23.16 & 174.39 & 68.90 \\
\hline Miscellaneous & 44.20 & & & 11.00 \\
\hline Disposal and landfill & & 4.53 & & 43.20 \\
\hline Total & 5043.26 & 41.19 & 174.39 & 4978.50 \\
\hline \multicolumn{5}{|l|}{ Rwanda (2005, HDI: 0.450) } \\
\hline Waste incineration & 8.57 & & & \\
\hline Ferrous and non-ferrous metal production & 2.40 & & & \\
\hline Power generation and heating & 6.48 & & & \\
\hline Production of mineral products & 0.60 & & & 0.10 \\
\hline Transport & 0.12 & & & \\
\hline Uncontrolled combustion processes & 46.60 & & & 14.55 \\
\hline Production and use of chemicals and consumer goods & & & 0.01 & \\
\hline Miscellaneous & 0.02 & & 0.02 & \\
\hline Disposal and landfill & & 1.00 & 0.05 & 41.50 \\
\hline Total & 64.80 & 1.00 & 0.09 & 56.15 \\
\hline
\end{tabular}


sources. Finally, the inventories from 51 countries are selected and analyzed.

\subsection{Data sources of national profiles}

Except for inventory data, national background profile data such as population and GDP are also necessary for this study. The NIPs provided country profiles of geography, population, GDP and so on. The fundamental information of the countries could also be cited from UN statistical common database (UN, 2007). Moreover, the Human Development Reports provided Human Development Index (HDI) in recent years. In this study, population, GDP and land area are the data of 2005, except for the HDI which is the data of 2006. GDP is converted into current US Dollars.

\section{Results and discussion}

\subsection{General state of national emissions}

\subsubsection{Statistical characteristics of total national emissions}

According to statistics, the selected 51 countries released about $36.0 \mathrm{~kg}$-TEQ dioxins $\mathrm{yr}^{-1}$ to the environment on average from 2000 to 2007. Pulles et al. (2006) reported an estimation of $3.3 \mathrm{~kg}$-TEQ dioxins $\mathrm{yr}^{-1}$ for 13 European countries, whose average national emission is about $1 / 3$ of the one for the 51 countries. The maximum and the minimum national emissions among these countries

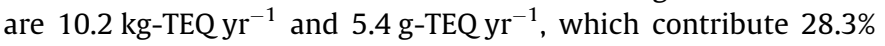
and $0.015 \%$ of the total emission, respectively. The maximum is 1800 times higher than the minimum implies large variance between the amounts of national emissions.

Fig. 1 shows the national total emissions and their cumulative proportion. In 51 countries, less than half of the countries contribute $90 \%$ of the total emission. Ten countries including China and Kenya have higher dioxin emission, contributing more than $70 \%$ of the total dioxins emission. These reflect that the national emissions vary significantly from country to country and minor number of countries dominates the total emission.

\subsubsection{Statistical characteristics of the categorized national emissions}

National emissions, classified by sources and media, are plotted in Fig. 2 by "Box-Whisker Chart" in Origin 7.5. The upper and lower “*” represent $1 \%$ and $99 \%$ outlier data points, respectively. The three horizontal lines in the box stand for $25 \%, 50 \%$, and $75 \%$ data sites, respectively, and " $\square$ " shows the position of the average. In Fig. $2 a$ and b, 5-95\% and $10-90 \%$ of the data are distributed between the two short horizontal lines, respectively.
From Fig. 2a and b, it could be seen that almost all the average emissions dependent on both the media and the source are between $75 \%$ and $90 \%$ data point. The average emissions of all these countries to air, water, products and soil/residue are 362, 14.1, 20.8 and $310{\mathrm{~g}-\mathrm{TEQ} \mathrm{yr}^{-1}}^{-1}$, respectively. The median halves $(25-75 \%)$ of the emission data to each medium are between 30 and 200, 0.2

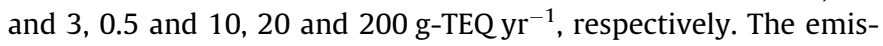
sions to air and soil/residue stay at a comparatively higher level. Two media accept $96.23 \%$ of the total emission with $53.41 \%$ to air and $42.82 \%$ to the soil/residue. It indicates that the dioxins are mainly released to air and soil/residue.

Among the nine main categories of the inventory, the uncontrolled combustion processes (UP) source is the biggest emission

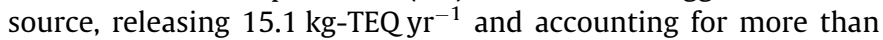
$40 \%$ of the total emission. This category could be divided into two subcategories, i.e. biomass burning, waste burning and accidental fires. Each subcategory contains several kinds of the subgroup sources, such as forest fires and uncontrolled domestic waste burning. The subgroup sources have large uncertainties in the emission estimation, resulting in rather significant deviations (Lemieux et al., 2004; Gullett et al., 2006; Pulles et al., 2006). This suggests that a single value could not precisely describe the actual situation, and some unpredictable factors should be considered during estimating. Grey system prediction theory was used to estimate the dioxins emission of "uncontrolled combustion processes" source (Zhang et al., 2008). This was an efficient attempt and could be considered as a new way to estimate the emission from this source.

Besides the uncontrolled combustion processes, waste incineration (WI), ferrous and non-ferrous metal production (FN) and power generation and heating (PG) are the three leading industrial sources for dioxins emissions, which contributed $13.2 \%$ (4.74 kgTEQ), $15.7 \%$ (5.64 kg-TEQ) and 16.3\% (5.85 kg-TEQ) of the total emission. The previous studies have mentioned this point of view (Anderson and Fisher, 2002; Breivik et al., 2004). These three sources are all typical industrial sources related with thermal processes, accounting totally for $45.1 \%$ of the total emissions. Such high contribution could suggest that the industrial thermal processes should be the key issue for the initial emission reduction (Japan, 2005).

\subsection{Correlations between emission and other factors}

With its uncontrollable, diffuse and stochastic properties (Breivik et al., 2004), the UP source is departed from the others by using principal component analysis. For further discussion, the total

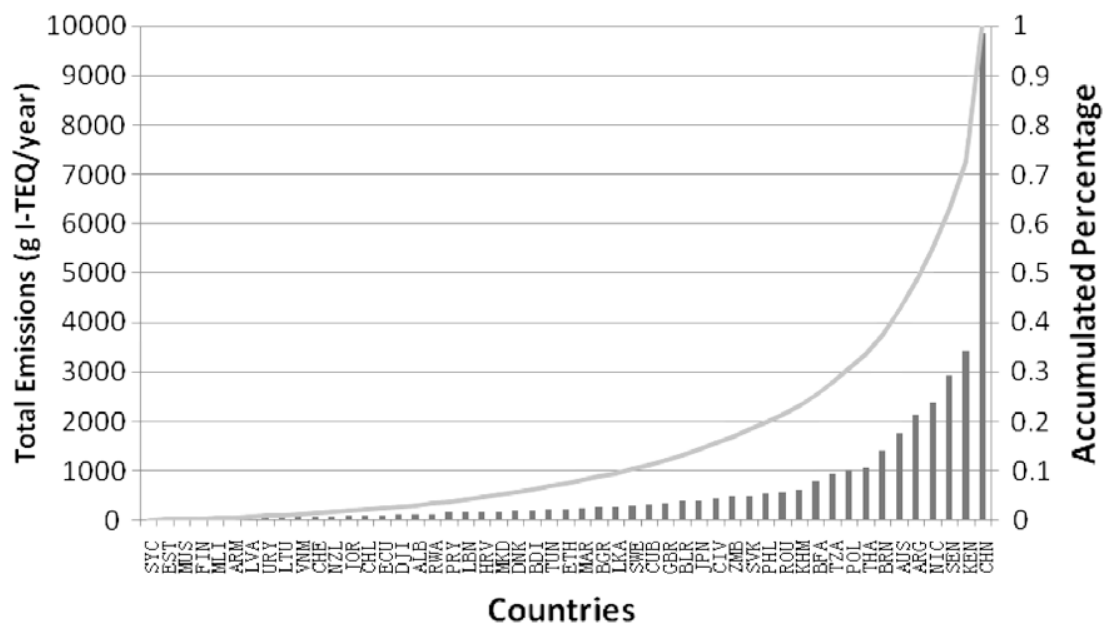

Fig. 1. National total emissions and cumulative proportion of the total emissions. Note: The country abbr. codes are cited from ISO3. 

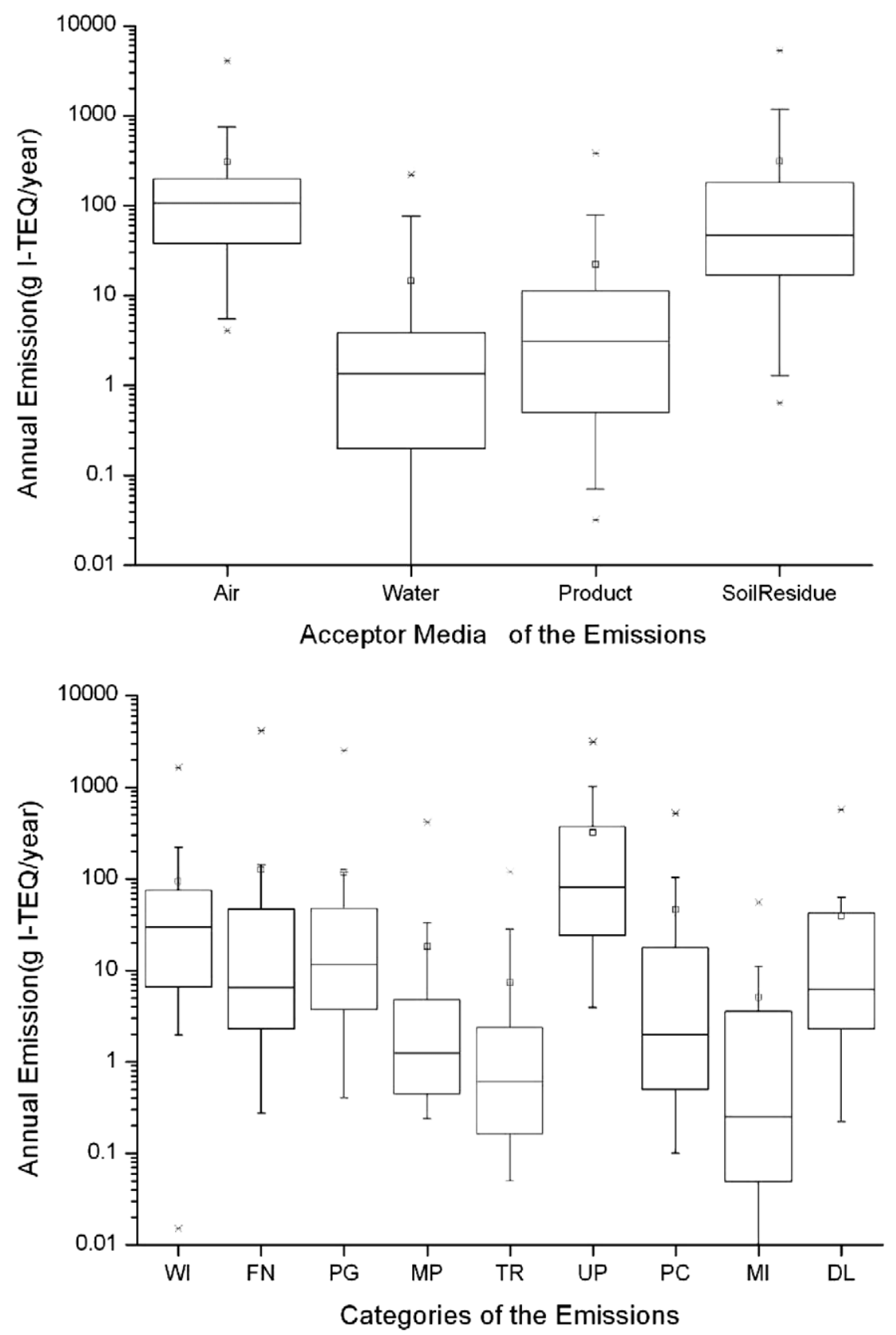

Fig. 2. (a) Dioxins released to different media (44 countries) and (b) annual categorical dioxins emissions (51 countries).

emission is divided into two groups, i.e. the emissions from the UP source and the total emission without the UP source (Total2).

Spearman correlation analysis was performed by using SPSS 13.0. Table 2 lists the correlation coefficients and their significant levels between emissions (UP, non-UP, total) and GDP/population/area/GDP per capita.

In the 51 countries, population (or area) has a very strong positive correlation with the total emission. Likewise, the correlations between UP (or Total2) emission and population (or area) are strongly positive. Population and land area provide the basis of human activities at national scale, e.g. natural resources, human resource and productions. Therefore, population and land area could be two positive factors of human activities to national emissions (Total, UP or Total2).

Total2 has a strong positive correlation with GDP. The major sources of Total2 are usually related with the industrial production processes, and industry is an important part of national economy which could be generally represented by GDP. Anyhow, GDP could be tentatively seen as a positive indicator for Total2.

There is a significant negative correlation between UP source and GDP per capita. GDP per capita is a sign for the nation's standard of living and the development extent of a country. It could be presumed from this negative correlation that higher development is helpful to reduce dioxins emissions from the UP source. Therefore, higher development might lead to less dioxins releases from the UP source, and GDP per capita could roughly be a negative indicator for UP source.

\subsection{Annual emissions per capita and per GDP}

As the emissions distribute diversely among countries, complex indicators are introduced to make further comparisons. Annual 
Table 2

Correlation coefficients between emissions (UP, non-UP, total) and GDP, population, area, GDP per capita $(N=51)$.

\begin{tabular}{|c|c|c|c|c|c|}
\hline Spearman's rho & & GDP & Population & Area & GDP per capita \\
\hline Total & Correlation coefficient & 0.227 & $0.577^{* *}$ & $0.422^{* *}$ & -0.218 \\
\hline UP & Correlation coefficient & -0.036 & $0.450^{* *}$ & $0.416^{* *}$ & $-0.423^{* *}$ \\
\hline Total2 & Correlation Coefficient & $0.465^{* *}$ & $0.544^{* *}$ & $0.349^{*}$ & 0.063 \\
\hline
\end{tabular}

Total2 (non-UP): the total emission except UP source.

* Correlation is significant at the 0.05 level (2-tailed)

** Correlation is significant at the 0.01 level (2-tailed).

emissions per billion-USD GDP (EPG) indicates the environmental cost for producing the unit value at national scale, while annual emissions per capita (EPP) represents the environmental pollution loading for each person in one country. The calculation formulas of annual emissions per unit GDP (EPG) and annual emissions per capita (EPP) are listed below:

$$
\begin{aligned}
& \mathrm{EPG}=\frac{\text { Annual Emissions }}{\text { National Gross Domestic Products }} ; \\
& \mathrm{EPP}=\frac{\text { Annual Emissions }}{\text { National Population }} .
\end{aligned}
$$

EPPs and EPGs of Total2 for the 51 countries are plotted in Fig. 3. Almost all the data points could be clustered into three groups - the six points below the line marked with 10000 USD person $^{-1}$ (Current US Dollar) and $1 \mathrm{~g}$-TEQ billion-USD ${ }^{-1}$ (excluding the four points inside the middle circle), the data concentrated in the middle circle field, and the four points above the lines marked with 1000 USD person $^{-1}$ and 100 g-TEQ billion-USD ${ }^{-1}$.

Switzerland, Finland, Norway, United Kingdom, Japan, and Netherlands are included in the first group. These countries are all highly developed countries and have taken actions to reduce dioxins emissions for many years. As a result, their GDP per capita are very high while they have very low EPGs. It hints that highly developed countries could reduce dioxins emissions to a very low EPG level.

The middle circle contains most of the countries which belong to the second group. Whether in the abscissa axis or in the vertical axis, the values wave between two orders of magnitude and comparatively evenly distribute in the circle. As the majority of these countries are on the beginning of reduction action, this group could represent the untamed state of dioxins emissions for most of the countries.

The third group is consisted of Nicaragua, Senegal, Sao Tome and Principe and Djibouti, which are mostly less developed countries (LDCs) with comparatively high emissions, EPP and EPG.

The most EPGs of the 51 countries are between 1 and 20 g-TEQ billion-USD ${ }^{-1}$, and their median and mean are 4.03 and $37.96 \mathrm{~g}$ TEQ billion-USD ${ }^{-1}$, respectively. Excluding the countries with extremely high EPGs (higher than 100, Nicaragua, Senegal, Sao Tome et Principe, Brunei, Djibouti) and the countries with rather low EPGs (lower than 0.5, Netherlands, Japan, UK, Sweden, Denmark, Finland, Switzerland), the median and the mean are recalculated as 4.12 and 6.75 g-TEQ billion-USD ${ }^{-1}$, respectively. These two values could better represent the average EPG for ordinary dioxins emission.

Likewise, the most EPPs of the 51 countries concentrated in the middle field are in the range of 1-100 g-TEQ million-people ${ }^{-1}$, and the median and mean of EPPs are calculated to be 9.5 and $14.5 \mathrm{~g}$ TEQ million-people ${ }^{-1}$, respectively.

In contrast to Fig. 3, the EPPs and EPGs of the UP source distribute dispersedly but evenly in the rectangle in Fig. 4. It implies an average distribution of the points along the contour and the perpendicular of GDP per capita in the rectangle area. Excluding those abnormal points outside the rectangle, the average EPP and EPG of these countries are calculated as follows. The results are $8.8 \mathrm{~g}$-TEQ

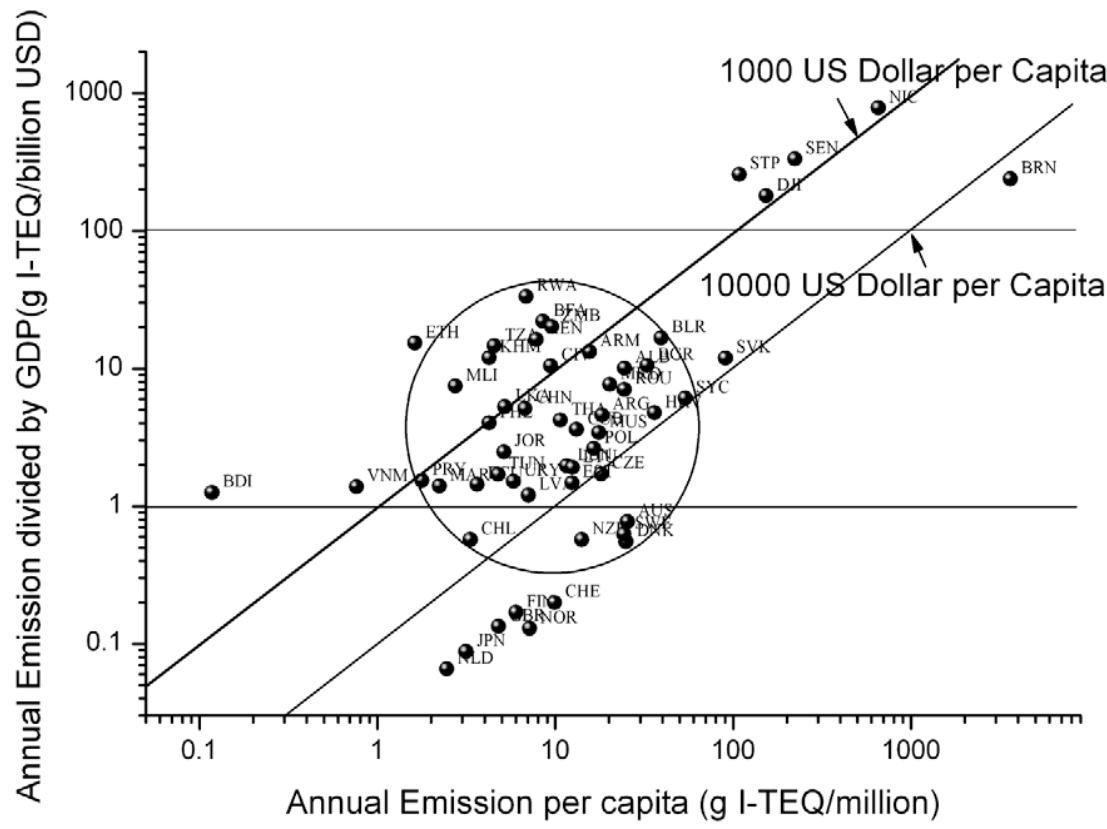

Fig. 3. Annual emissions per capita and per unit GDP of each country. Note: Here, the total emission is without uncontrolled combustion processes source. 


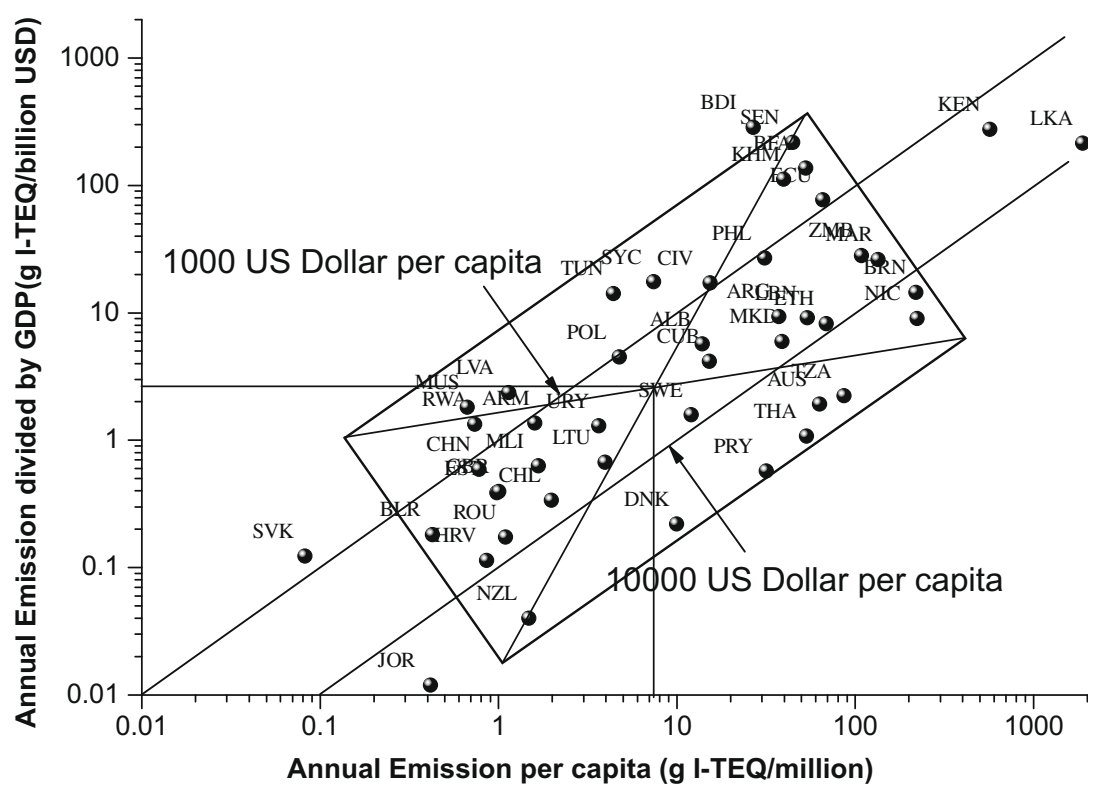

Fig. 4. Dioxins emissions of UP source by per capita and per unit GDP.

million-people ${ }^{-1}$ for EPP and 2.6 g-TEQ billion-USD ${ }^{-1}$ for EPG, respectively

$\mathrm{EPP}_{\text {Average Level }}=10^{\frac{1}{n} \sum \log _{10} \mathrm{EPP}}$ and $\quad \mathrm{EPG}_{\text {Average Level }}=10^{\frac{1}{n} \sum \log _{10} \mathrm{EPG}}$.

According to Figs. 3 and 4, the EPPs and EPGs of the total emissions are adding up to 18.3 g-TEQ million-people ${ }^{-1}$ and 6.7 g-TEQ billion$\mathrm{USD}^{-1}$ on average, respectively. The results are comparable with a median of EPPs as $21 \mathrm{~g}$-TEQ million-people ${ }^{-1}$ based on the inven- tory data from 23 countries reported by Fiedler (2007). Since the extreme high or low points are excluded in this study, the results could better represent the average EPPs and EPGs at national scale.

\subsection{Dioxins emission and human development}

The HDI (Human Development Index) is a summary composite index that measures a country's average achievements in three basic aspects of human development: health, knowledge, and a de-
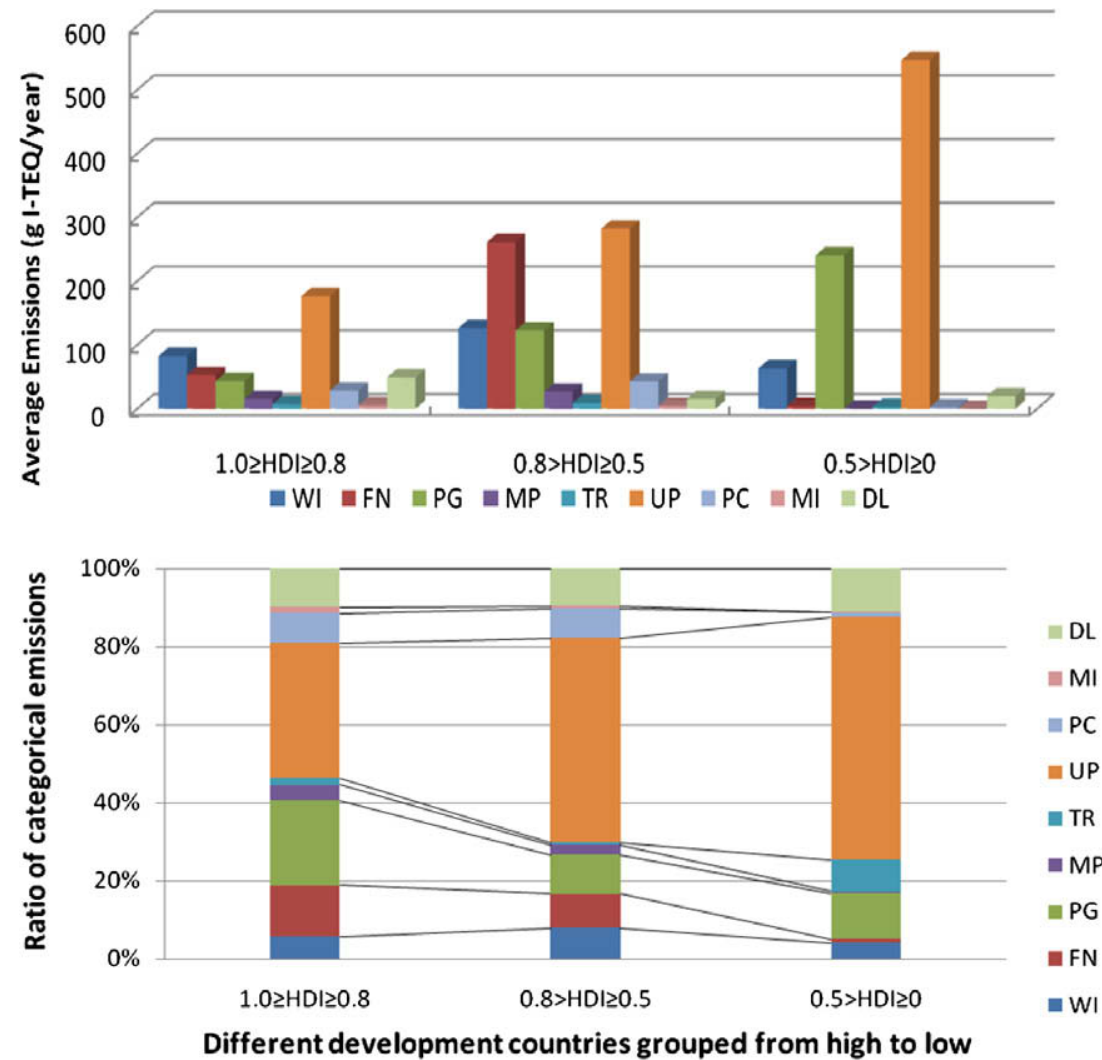

Fig. 5. National average dioxins emissions and their ratios in different HDI groups. 
cent standard of living. In the Human Development Report, HDI between 1.0 and 0.8 represents high human development, while HDI between 0.8 and 0.5 means medium human development and HDI below 0.5 stands for low human development (UNDP, 2006).

Comparing the average emissions among the different HDI groups, it can be seen from Fig. 5 that emission of UP is high in all HDI groups; the emissions of WI, FN and PG are relatively high in the high and medium HDI groups; the low HDI group has a significantly high emission from UP and PG, while its emissions from the other sources are very low.

Due to the efforts of dioxins reduction during the past years, the emissions of the industrial sources are greatly reduced in the high HDI countries, and some other sources like PG came out to be the new leading emission sources instead. And for those developing countries mostly with medium HDIs, the typical "high energy consumption and high environmental pollution" developing style seems to be inevitable, and their industrial sources release more dioxins than that of emissions in less developed countries or highly developed countries. As listed in Table 1, three national inventories in different HDI groups represent three types of distributions of categorized dioxins emissions.

\section{Conclusions}

In the current study, the national dioxins emission inventories of 51 countries have been statistically analyzed combined with some profile factors. Between 2000 and 2007, the average national dioxins emission of the 51 countries is $0.71 \mathrm{~kg}^{-T E Q ~} \mathrm{yr}^{-1}$. For the 51 countries, the diverse emission levels imply that minor countries with the largest emissions dominate the total dioxins emission. Furthermore, the total emission per capita and the total emission per billion-USD are calculated to be $18.3 \mathrm{~g}$-TEQ million-people ${ }^{-1}$ and 6.7 g-TEQ billion-USD ${ }^{-1}$ on average, respectively. These composite indicators could characterize the national emissions from two aspects of human activities and better reflect the situations of dioxins emissions of the countries.

Waste incineration, ferrous and non-ferrous metal production and power generation and heating are the three leading industrial emission sources, contributing $45.1 \%$ to the total emissions. Industrial production could be considered as a major group of human activities that release dioxins to the environment. Besides, domestic emission sources, partly including uncontrolled combustion processes, are also an important group of emission sources that are worthy of being focused.

For the 51 countries, population and land area have positive correlations with national dioxins emissions. As a factor of human activities, population is not only an indicator of productivity, but also the base of consumption. In both aspects, the dioxins emissions are involved. As a result, population is tentatively suggested to be a positive indicator of national dioxins emission. Moreover, there is a positive correlation between GDP and total emission excluding UP source. In contrast, the correlation between GDP per capita and emission of UP source is negative. Hereby, GDP and GDP per capita could be roughly regarded as two indicators for the categorized national dioxins emissions. Moreover, the ratio structure of the categorized emissions could be probably influenced by HDI. There are some similarities of the dioxins emissions among the countries at the same HDI level.

\section{Acknowledgements}

This study was supported by Chinese Academy of Sciences (Grant No. KZCX2-YW-420), National 973 Program (2009CB421606) and National Natural Science Foundation of China (20621703).

\section{References}

Alcock, R.E., Sweetman, A.J., Jones, K.C., 2001. A congener-specific PCDD/F emissions inventory for the UK: Do current estimates account for the measured atmospheric burden? Chemosphere 43, 183-194.

Anderson, D.R., Fisher, R., 2002. Sources of dioxins in the United Kingdom: the steel industry and other sources. Chemosphere 46, 371-381.

Bhavsar, S.P., Reiner, E.J., Hayton, A., Fletcher, R., MacPherson, K., 2008. Converting toxic equivalents (TEQ) of dioxins and dioxin-like compounds in fish from one toxic equivalency factor (TEF) scheme to another. Environment International 34, 915-921.

Breivik, K., Alcock, R., Li, Y.F., Bailey, R.E., Fiedler, H., Pacyna, J.M., 2004. Primary sources of selected POPs: regional and global scale emission inventories. Environmental Pollution 128, 3-16.

Breivik, K., Vestreng, V., Rozovskaya, O., Pacyna, J.M., 2006. Atmospheric emissions of some POPs in Europe: a discussion of existing inventories and data needs. Environmental Science \& Policy 9, 663-674.

Douben, P.E.T., 1997. PCDD/F emissions to atmosphere in the UK and future trends. Chemosphere 34, 1181-1189.

Dyke, P.H., Foan, C., Wenborn, M., Coleman, P.J., 1997. A review of dioxin releases to land and water in the UK. Science of the Total Environment 207, 119-131.

Eduljee, G.H., Dyke, P., 1996. An updated inventory of potential PCDD and PCDF emission sources in the UK. Science of the Total Environment 177, 303-321.

Fiedler, H., 2007. National PCDD/PCDF release inventories under the Stockholm convention on persistent organic pollutants. Chemosphere 67, S96-S108.

Fiedler, H., Hutzinger, O., 1992. Sources and sinks of dioxins - Germany. Chemosphere 25, 1487-1491.

Gribble, G.W., 1996. Dioxin inventory. Environmental Science \& Technology 30, A184.

Gullett, B.K., Touati, A., Huwe, J., Hakk, H., 2006. PCDD and PCDF emissions from simulated sugarcane field burning. Environmental Science \& Technology 40 , 6228-6234.

Harrad, S.J., Jones, K.C., 1992. A source inventory and budget for chlorinated dioxins and furans in the United-Kingdom environment. Science of the Total Environment 126, 89-107.

Japan, 2005. The National Implementation Plan of Japan under the Stockholm Convention on Persistent Organic Pollutants.

Jurado, E., Jaward, F.M., Lohmann, R., Jones, K.C., Simo, R., Dachs, J., 2004 Atmospheric dry deposition of persistent organic pollutants to the Atlantic and inferences for the global oceans. Environmental Science \& Technology 38 5505-5513.

Lemieux, P.M., Lutes, C.C., Santoianni, D.A., 2004. Emissions of organic air toxics from open burning: a comprehensive review. Progress in Energy and Combustion Science 30, 1-32.

Pulles, T., Kok, H., Quass, U., 2006. Application of the emission inventory model TEAM: uncertainties in dioxin emission estimates for central Europe. Atmospheric Environment 40, 2321-2332.

Quass, U., Fermann, M., Broker, G., 2004. The European dioxin air emission inventory project - final results. Chemosphere 54, 1319-1327.

Sheffield, A., 1985. Sources and releases of PCDD's and PCDF's to the Canadian environment. Chemosphere 14, 811-814.

Stockholm Convention, 2001. Stockholm Convention on Persistent Organic Pollutants. <http://www.pops.int/>.

UK, 2007. National Implementation Plan for the Stockholm Convention on Persistent Organic Pollutants.

UN, 2007. United Nations Common Database, New York, USA <http:// www.unstats.un.org/unsd/default.htm>.

UNDP, 2006. Human Development Reports. <http://www.hdr.undp.org/en/ statistics/>.

UNEP, 2000-2003. National PCDD/PCDF Inventories. <http://www.chem.unep.ch/ pops/pcdd_activities/inventories/default.htm>.

UNEP, 2005. Standardized Toolkit for Identification and Quantification of Dioxin and Furan Release, UNEP Chemicals, Geneva, Switzerland <http:// www.chem.unep.ch/pops/pcdd_activities/toolkit/default.htm>.

Van der Gon, H.D., Van het Bolscher, M., Visschedijk, A., Zandveld, P., 2007. Emissions of persistent organic pollutants and eight candidate POPs from UNECE-Europe in 2000, 2010 and 2020 and the emission reduction resulting from the implementation of the UNECE POP protocol. Atmospheric Environment 41, 9245-9261.

Wittsiepe, J., Fürst, P., Wilhelm, M., 2007. The 2005 World Health Organization reevaluation of TEFs for dioxins and dioxin-like compounds - What are the consequences for German human background levels? International Journal of Hygiene and Environmental Health 210, 335-339.

Zhang, Q., Huang, J., Yu, G., 2008. Polychlorinated dibenzo-p-dioxins and dibenzofurans emissions from open burning of crop residues in China between 1997 and 2004. Environmental Pollution 151, 39-46. 